\title{
Aprimorando um Jogo para Ensino do Método de Avaliação de Comunicabilidade por Meio de Estudos Experimentais
}

\author{
Ruddá Silva Beltrão Brito ${ }^{1}$, Martha Silva Fernandes ${ }^{2}$, Wagner Queiroz Junior ${ }^{1}$, \\ Bruno A. Bonifácio ${ }^{1}$, Priscila S. Fernandes ${ }^{1,3}$ \\ ${ }^{1}$ Instituto de Ciências Exatas e Tecnologia (ICET) - Universidade Federal do Amazonas \\ (UFAM) - Caixa Postal 15.064 - 91.501-970 - Itacoatiara - AM - Brazil \\ ${ }^{2}$ Instituto Federal do Amazonas (IFAM) - Caixa Postal 100.665 - Manaus - AM-Brazil \\ ${ }^{3}$ Grupo de Usabilidade e Engenharia de Software (USES) - \\ Universidade Federal do Amazonas (UFAM)

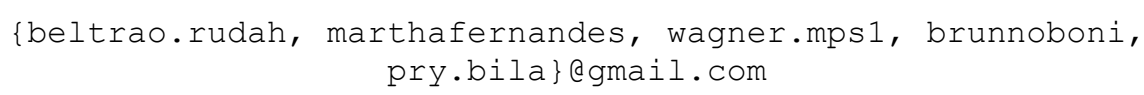

Abstract.The popularity of tablets and smartphones, enabled the emergence of increasingly interactive applications. For this reason, the software development process also needed to evolve in order to meet users' expectations, new knowledge, techniques and tools that go beyond the methods of Software Engineering. In this context, the Human-Computer Interaction area (HCI) brings an innovative insight into the process of creating applications in technological education centers. However, teach IHC is not easy. For this reason, use new teaching approaches can help in more collaborative educational activities. This article presents a teaching approach of one of the important concepts of IHC, as Semiotic Engineering. The MACteaching uses simulation to teach the Communicability Evaluation Method (CEM) as a way to encourage students, creating a more dynamic environment. Preliminary results show significant acceptance when using MACteaching as a complementary approach to education.

Resumo. A popularização de dispositivos móveis possibilitou o surgimento de aplicações cada vez mais interativas. Por essa razão, o processo de desenvolvimento de software também precisou evoluir, para atender as expectativas dos usuários com novos conhecimentos, técnicas e ferramentas. Nesse contexto, a área Interação Humano-Computador (IHC) traz uma visão inovadora sobre o processo de criação de aplicativos nos centros de ensino tecnológico. Todavia ensinar IHC não é tarefa fácil. Por essa razão, utilizar novas abordagens de ensino pode auxiliar em atividades pedagógicas mais colaborativas. Este artigo apresenta uma abordagem de ensino de um dos conceitos importantes de IHC, a Engenharia Semiótica. A abordagem consiste em um jogo especifico para o contexto móvel, o MACteaching. O jogo utiliza simulação para o ensino do método de avaliação de comunicabilidade (MAC), como forma de estimular os estudantes, criando um ambiente mais dinâmico. Os resultados experimentais mostram importante aceitação ao utilizar o MACteaching como abordagem complementar de ensino. 


\section{Introdução}

A combinação entre tecnologias móveis e os serviços da Internet alteraram o modo como os usuários interagem e acessam as informações [Coutinho et al. 2014]. Essa transformação impulsionou o surgimento de novas formas de interação e aprendizado, criando os mais variados perfis de usuários que utilizam dispositivos móveis. Devido essa diversidade, surge a necessidade de criação de aplicativos cada vez mais interativos, para atender os mais diversos perfis de usuários que interagem com essas aplicações [Meireles; Bonifácio 2015]. Porém, saber como criar novas aplicações, que atendam as expectativas dos usuários, exige conhecimento, técnicas e ferramentas que vão além dos métodos da Engenharia de Software (ES).

Nesse contexto, a área Interação Humano-Computador (IHC) traz uma visão inovadora sobre o processo de criação de aplicativos, considerando fatores focados nos usuários e como atender de maneira satisfatória suas necessidades e expectativas [Benitti e Sommariva 2012]. Assim, a área de IHC tem evoluído para propiciar aos pesquisadores e desenvolvedores um corpo de conhecimento sobre os novos contextos de uso e requisitos para construção de interfaces [Valentim et al. 2014]. Devido essas características o ensino de IHC tem sido cada vez mais difundido em cursos de graduação e pós-graduação, propiciando a formação de profissionais mais qualificados, e consequentemente, sistemas computacionais de melhor qualidade. No entanto, a natureza interdisciplinar da área, que envolve várias disciplinas e métodos de avaliação de aplicações constitui importantes questões de pesquisa [Bim et al. 2012].

Para lidar com esses desafios, professores tem se esforçado para criar um ambiente motivador, no qual seja possível ensinar os estudantes e profissionais os fundamentos de IHC, por meio de materiais de ensino, exemplos e dinâmicas para aplicar durante suas aulas. Utilizar tecnologias móveis, como recurso complementar, no processo de ensino pode auxiliar na criação de atividades pedagógicas mais colaborativas e inovadoras. Assim, utilizar abordagem de jogos educativos como abordagem de ensino pode auxiliar no desenvolvimento de habilidades cognitivas a resolução de problemas, tomada de decisão, reconhecimento de padrões, processamento de informações, criatividade e pensamento crítico, e com isso potencializar o aprendizado, possibilitar a fixação do conteúdo de forma mais eficaz e incentivar o aprendizado através de desafios.

Esse cenário motivou a elaboração do jogo para dispositivos móveis, chamado MACteaching. Essa solução utiliza uma abordagem de interação que visa melhorar a experiência de alunos no ensino de IHC, dos cursos de Engenharia de Software e Sistemas de Informação, especificamente no ensino de um método fundamentado na Engenharia Semiótica, o Método de Avaliação de Comunicabilidade (MAC). A escolha do MAC é justificada devido à dificuldade que normalmente os alunos apresentam no ensino de algumas etapas do método, o que constitui grande oportunidade de pesquisa. O MACteaching utiliza simulações para reforçar os conceitos do MAC.

O objetivo desse artigo consiste em: 1) apresentar o as características que tornam o jogo uma solução que pode contribuir para o processo de ensino-aprendizagem em IHC e ES; além de 2) relatar os estudos realizados até o momento para avaliar o jogo e; 3 ) as melhorias identificadas para aprimorar a experiência de uso do aplicativo. Esperase com isso, auxiliar no ensino de métodos que possam enriquecer o ensino na área de IHC para ajudar na produção de aplicativos com maior qualidade de uso. 
O artigo está organizado da seguinte forma: A Seção 2 descreve os conceitos e trabalhos relacionados à Engenharia Semiótica, o MAC e os jogos para ensino de IHC. A Seção 3 apresenta o jogo MACteaching. A Seção 4 apresenta a metodologia utilizada para realizar o estudo, a condução da avaliação realizada, e também discute os resultados obtidos. A Seção 5 apresenta a análise e dificuldades. Por fim, a Seção 6 lista as conclusões e trabalhos futuros.

\section{Conceitos e Trabalhos Relacionados}

O aparecimento constante de novas tecnologias tem alterado a forma como os usuários interagem com os sistemas interativos; e consequentemente como as tecnologias de software são desenvolvidas. Todavia, desenvolver novos produtos, avaliá-los, e saber quando avaliar são desafios para área de IHC. Vários métodos para avaliar a qualidade de sistemas têm sido propostos. Dentre estes, estão aqueles fundamentados na Engenharia Semiótica.

\subsection{Engenharia Semiótica}

A Engenharia Semiótica é uma teoria de IHC que caracteriza a interação usuáriosistema como um tipo específico de comunicação, categorizando aplicações computacionais como artefatos de metacomunicação [Bim et al. 2011]. A metamensagem ocorre na comunicação existente entre usuário e designer, por meio da interface [Savi e Ravi 2008]. A Inspeção Semiótica e o Método de Avaliação de Comunicabilidade (MAC) são exemplos de métodos de avaliação fundamentados na Engenharia Semiótica.

\subsection{Método de Avaliação de Comunicabilidade}

Devido sua relevância o MAC tem sido amplamente adotado [Prates e Barbosa 2007]. Este método tem como objetivo avaliar a qualidade da metacomunicação entre o designer e o usuário. De acordo com [Barbosa e Silva 2010], o método possui cinco atividades: (1) preparação, onde devem ser realizadas tarefas como definir tarefas a serem executadas pelos participantes, definir o perfil e selecionar os participantes; (2) coleta de dados, onde os usuários são observados e suas sessões são registradas; (3) interpretação, onde é feita a etiquetagem dos vídeos etiquetas definidas no MAC; (4) consolidação dos resultados, onde é realizada interpretadas as etiquetagens e elaborar o perfil semiótico; e (5) relato dos resultados. Como o MAC possui um total de treze etiquetas as quais possuem contextos específicos, no processo de ensino, os estudantes comumente encontram dificuldade para utilização do método.

\subsection{Trabalhos Relacionados}

Jogos de ensino de IHC têm sido propostos na literatura nos últimos anos. Como propõe [Sommariva et al. 2011] a aula se torna mais descontraída, tendo em vista que o jogo proporciona aos alunos um ambiente livre de riscos. Conseguindo realizar ainda uma rápida troca de problemáticas, a qual não é possível em ambientes não virtuais. A Tabela 1 apresenta alguns destes trabalhos. 
Tabela 1.Trabalhos Relacionados

\begin{tabular}{|c|c|c|l|}
\hline Autor(es) & $\begin{array}{c}\text { Aplicado } \\
\text { para o ensino } \\
\text { de IHC }\end{array}$ & Descrição & Conceito Aplicado \\
\hline $\begin{array}{c}\text { [Sommariva } \\
\text { et al. 2011] }\end{array}$ & Sim & $\begin{array}{c}\text { Propõe um jogo de avaliação, } \\
\text { específico para aplicativos móveis. } \\
\text { E utiliza como base a avaliação } \\
\text { heurística }\end{array}$ & $\begin{array}{l}\text { 1. Analogia } \\
\text { 2.Heurísticas de } \\
\text { Nielsen }\end{array}$ \\
\hline $\begin{array}{c}\text { [Ferreira et } \\
\text { al. 2014] }\end{array}$ & Sim & $\begin{array}{c}\text { Propõe um jogo para o ensino de } \\
\text { avaliação de usabilidade aplicado } \\
\text { usando a avaliação heurística. }\end{array}$ & $\begin{array}{l}\text { 1.Heurísticasde } \\
\text { Nielsen 2.Casos de } \\
\text { uso em avaliações }\end{array}$ \\
\hline $\begin{array}{c}\text { [Cardoso et } \\
\text { al. 2007] }\end{array}$ & Não & $\begin{array}{c}\text { Propõe uma ferramenta de apoio do } \\
\text { MAC. Aplicado para avaliação de } \\
\text { interfaces web }\end{array}$ & $\begin{array}{l}\text { 1.MAC 2.Avaliação } \\
\text { de interfaces }\end{array}$ \\
\hline $\begin{array}{c}\text { [Shimada et } \\
\text { al. 2015] }\end{array}$ & Sim & $\begin{array}{c}\text { Utiliza jogos digitais para o ensino } \\
\text { de IHC. }\end{array}$ & $\begin{array}{l}\text { 1.Conceitos de IHC } \\
\text { 2. Métodos de } \\
\text { avaliação de } \\
\text { usabilidade }\end{array}$ \\
\hline
\end{tabular}

Apesar das iniciativas para o desenvolvimento de jogos para o ensino de IHC, a literatura apresenta poucos trabalhos específicos para ensino de conceitos de Engenharia Semiótica, o mesmo para o ensino do o MAC. Por essa razão, optou-se por desenvolver um jogo que auxiliasse no ensino do MAC. A próxima Seção apresenta o funcionamento do jogo.

\section{MACteaching}

O jogo educativo MACteaching tem como proposta facilitar o processo de ensino e aprendizagem do MAC. O MACteaching utiliza como abordagem interativa de aprendizagem relacional, por meio de associação por analogia. O propósito do jogo é ser uma ferramenta complementar ao ensino do método, focando em trabalhar os conceitos de forma lúdica. O jogo caracteriza-se como um simulador prático dos conceitos do MAC, pois apresenta cenários descrevendo casos de uso que são passíveis de acontecer durante a observação do participante, na aplicação do método. Além de prática, com exercícios que favorecem o ensino das etapas do método, apresentando de maneira rápida, novas problemáticas, para que o jogador possa se familiarizar rapidamente com as etiquetas e os conceitos da área. $\mathrm{O}$ jogo foi desenvolvido para plataforma Android.

A plataforma de desenvolvimento utilizada foi o Android Studio, para criação e edição de imagens foi usado o Photoshop CS. A interface do jogo MACteaching, apresentado na Figura 1, foi organizada da seguinte maneira: ao iniciar o jogo, o aluno é inserido na história do jogo, onde são apresentados os conceitos da área por meio de analogias e personagens. A história apresentada no jogo é centrada em uma cidade, chamada Interacionópolis, onde vivem desenvolvedores e designers produzindo 
sistemas. Porém, surge uma bruxa, chamada Ruptura, que se incomoda com o sucesso dos desenvolvedores e começa a inserir várias rupturas de interação nas interfaces dos sistemas gerados dentro da cidade.

Para resolver a situação, uma equipe de desenvolvimento resolve aplicar o Método de Avaliação de Comunicabilidade (MAC). O jogo então tem o propósito de identificar as rupturas nos sistemas, onde o jogador do aplicativo atua como personagem principal, um participante chamado Meckinho. O objetivo é identificar, para cada ruptura qual a etiqueta do método que expressa o problema de comunicação na interface gráfica dos sistemas interativos.
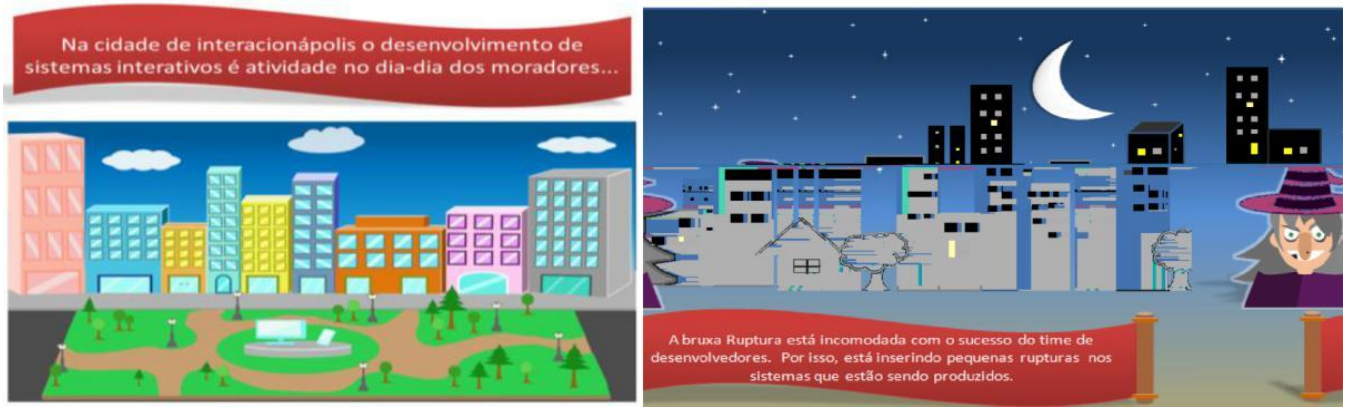

Figura 1. Narrativa do Jogo MACteaching.

Para a interação realizada pelo jogador durante a simulação, foi utilizado um mecanismo de drag-and-drop, Figura 2. O objetivo do jogo é conseguir etiquetar o máximo de rupturas corretamente e salvar a cidade da bruxa Ruptura. O jogo disponibiliza ainda descrições das treze etiquetas do MAC, com interface intuitiva e amigável. A versão inicial do jogo possui um nível iniciante e intermediário até o momento.

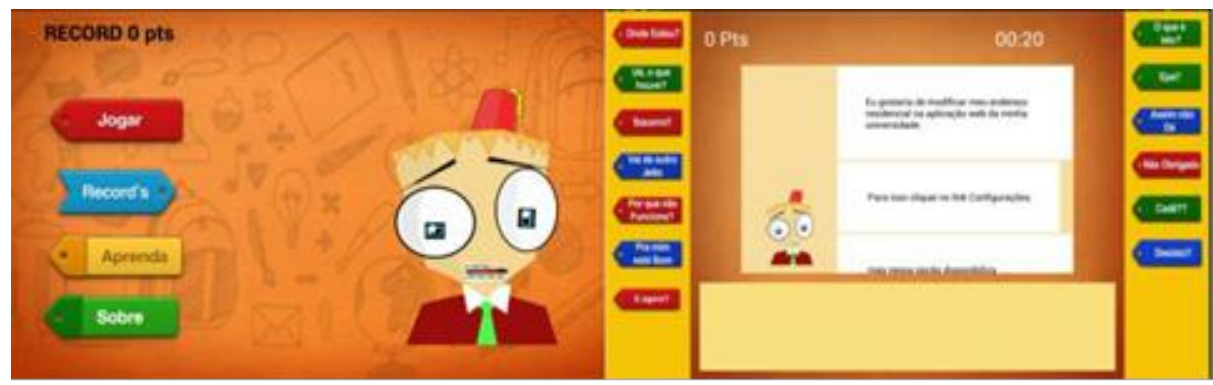

Figura 2. Interface gráfica do jogo.

\section{Avaliando e Aprimorando o Jogo por Meio de Estudos Experimentais}

Para entender a aplicabilidade do jogo MACteaching, como ferramenta capaz de estimular e contribuir com a construção de conhecimento e habilidades dos estudantes em relação ao $\mathrm{MAC}$, foram feitos até o momento dois estudos experimentais. No primeiro buscou avaliar a usabilidade do jogo em relação a três aspectos de interação: a utilidade percebida, a facilidade de uso e a contribuição para o aprendizado. O Segundo estudo foi conduzido através de um estudo de observação, de forma qualitativa e quantitativa a contribuição do aplicativo no ensino do MAC. Para isso, foi realizado inicialmente um estudo piloto, com discentes do Curso de Sistemas de Informação em 
uma universidade no norte do país. Estes estudos serão apresentados a seguir.

\subsection{Primeiro Estudo Experimental}

O primeiro estudo realizado no MACteaching foi um teste de usabilidade, cujo objetivo foi avaliar a qualidade de interação do aplicativo e a percepção de facilidade de uso.

Seleção dos Participantes: Para este estudo foram selecionados seis alunos de graduação em Informática. Os participantes foram divididos considerando os níveis de experiência em IHC e conhecimento no método MAC. Objeto de estudo: versão inicial do MACteaching.

Execução: Após o uso do aplicativo, foram feitas entrevistas e aplicados questionários de avaliação sobre a experiência de uso e aprendizado dos participantes com o MACteaching. Os resultados foram agrupados em pontos fortes, pontos a serem melhorados e a percepção do participante ao usar o aplicativo como ferramenta de apoio ao ensino do Método de Avaliação de Comunicabilidade.

Resultados Obtidos: Como resultado da avaliação inicial foram identificados cinco problemas de usabilidade. Após a etapa de execução do estudo, foram coletadas as informações as quais geraram gráficos demonstrativos agrupando informações a respeito da facilidade de uso, aprendizagem do Método de Avaliação de Comunicabilidade e sugestões e melhorias para o MACteaching. As escalas foram baseadas no Modelo de Aceitação de Tecnologia, para o uso de escalas de likert, que permitiram melhor análise quantitativa acerca dos resultados obtidos, conforme apresentado na Figura 3.

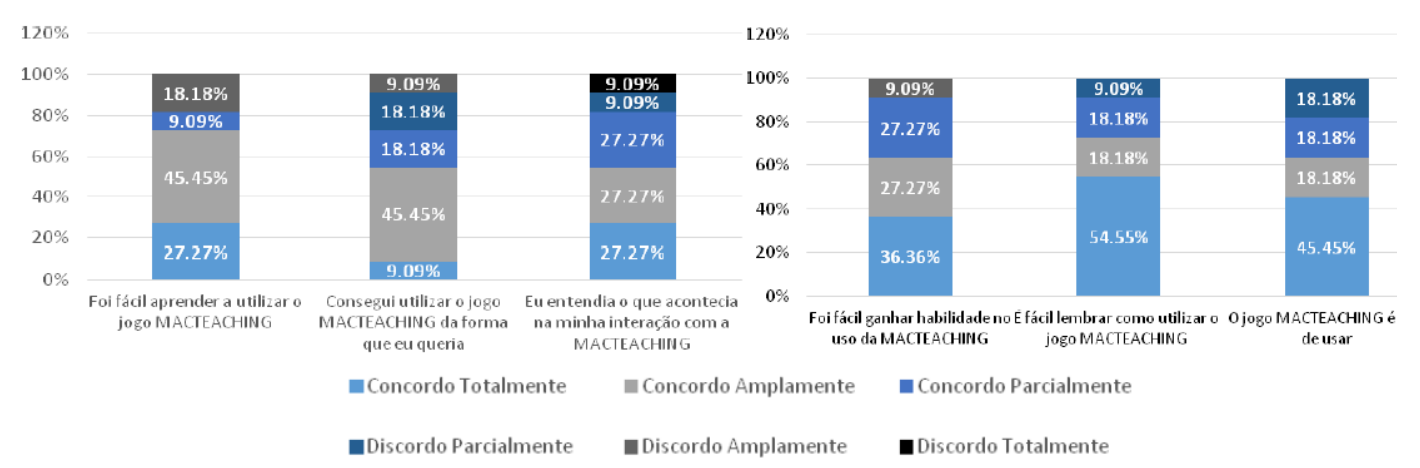

Figura 3. Resultado do Primeiro Estudo Experimental

O estudo também indicou os pontos fortes e de melhoria do aplicativo. Em relação aos pontos fortes, pode-se citar, o aluno 1 que afirmou: "A aplicação é uma ótima ferramenta para o aprendizado e assimilação da técnica $M A C$ ", o aluno 4: "É iterativo, deixando o usuário com vontade de querer jogar" e o aluno 5: "Aprendi onde devo utilizar as etiquetas". Outro ponto observado, durante a avaliação, foi que a maioria dos alunos mencionou a aprendizagem do MAC como ponto forte do jogo.

No aspecto de sugestões e melhorias, a maioria dos alunos se sentiram prejudicadas pela falta de feedback da aplicação em relação as suas respostas, no jogo. Podemos destacar, a afirmação do aluno 1 que relatou: "A aplicação deveria informar ao usuário uma mensagem informando que o mesmo selecionou a resposta incorreta", pelo aluno 2 "Falta de resposta ao erro", e do aluno 4, que relatou: "Não aparecem 
mensagens quando erramos". Além disso, foram observadas dificuldades dos participantes na interação com o aplicativo, principalmente para mover as etiquetas do método. Outro ponto mencionado foi a disputa com outros jogadores, que pode tornar o jogo mais atrativo.

Assim, a principal contribuição desse estudo inicial foi a identificação de pontos de melhoria na interface gráfica do aplicativo. Foi identificado um total de cinco pontos de melhoria, como: dificuldade em saber para onde arrastar as etiquetas, a velocidade da história inicial do jogo, dificuldade de leitura em legendas do jogo; feedbacks do jogo sem destaque e; tempo de jogo muito curto. $O$ estudo serviu de base para alteração na interface gráfica do aplicativo, como apresentado na Figura 4.
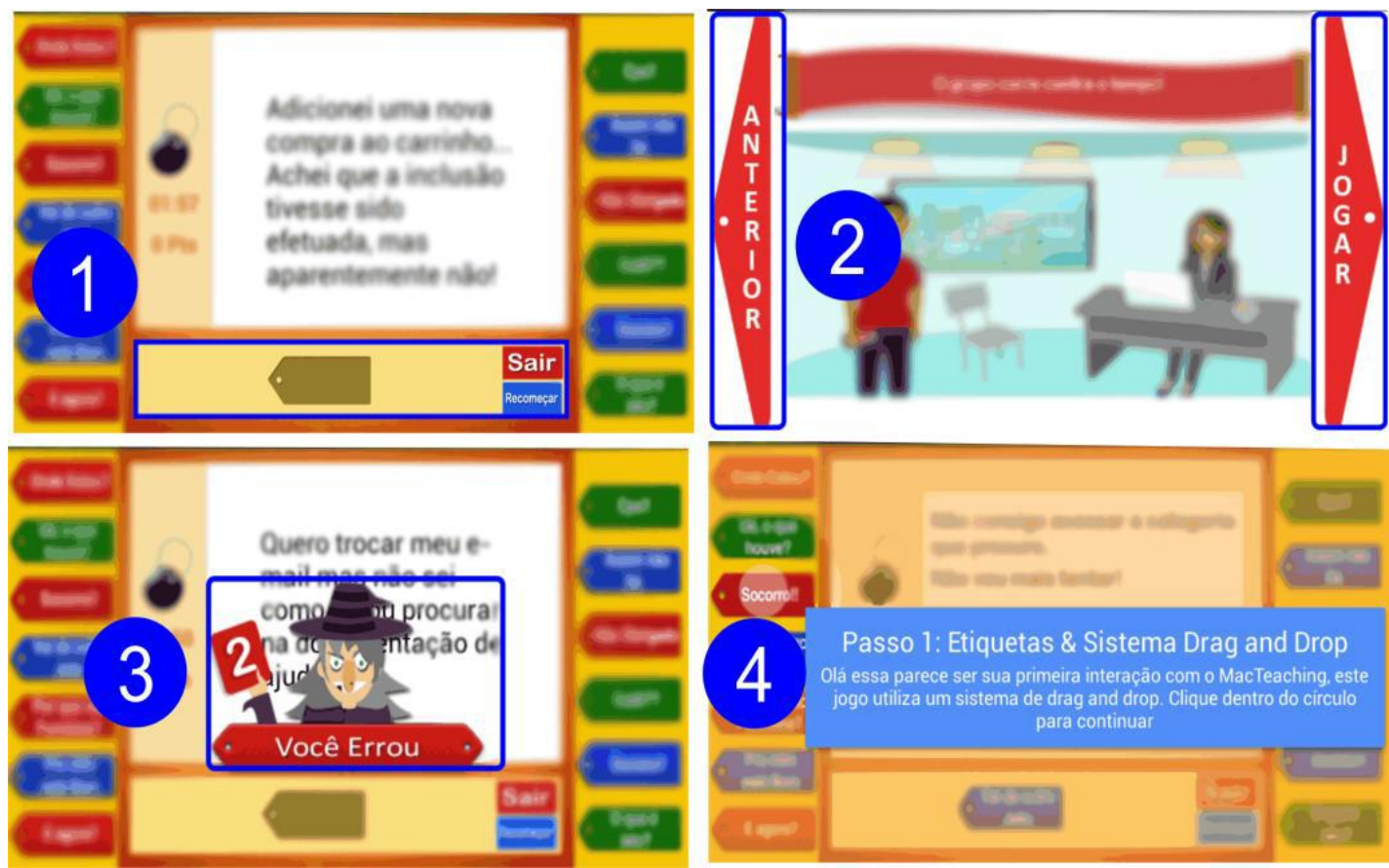

\section{Figura 4. Alterações realizadas na interface gráfica do aplicativo após o estudo.}

A Figura 4 apresenta as melhorias realizadas no aplicativo tais como: indicação na interface gráfica do aplicativo mostrando o local de movimento das etiquetas (Figura 5-1); novos botões de controle avançar/jogar e voltar para melhor leitura da historinha do jogo (Figura 5-2); feedback após interação do jogo, com imagens que ilustram a resposta do usuário (Figura 5-3); aumento do tempo após iniciar a partida, além de ranking dos 10 (dez) melhores jogadores. E por fim, um tutorial sobre o funcionamento do jogo, mostrando instruções e pontos que auxiliam no aprendizado do jogo e do MAC (Figura 5-4).

\subsection{Segundo Estudo Experimental}

Embora o primeiro estudo experimental mostre indícios da contribuição do MACteaching para o ensino do MAC, o primeiro estudo consistia apenas em obter melhorias na usabilidade do aplicativo. Dessa forma, foi elaborado um estudo de 
observação com o objetivo de entender como os usuários utilizam o jogo, como objeto de aprendizagem complementar.

Preparação: $O$ propósito do estudo foi avaliar, de forma qualitativa a contribuição do aplicativo no ensino do MAC. Para isso, foi realizado um estudo, com discentes do Curso de Sistemas de Informação em uma universidade no norte do país. Assim, como o primeiro estudo foi utilizado como indicador dois aspectos de interação: a utilidade percebida e a contribuição para o aprendizado. Para a condução do estudo, foi elaborado um conjunto de etapas, baseado em [Valentim et al. 2014] apresentado na Tabela 2.

Tabela 2. Fases do Planejamento Experimental

\begin{tabular}{|c|c|c|}
\hline Etapa & Descrição & Etapa \\
\hline \multirow{4}{*}{ 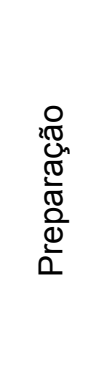 } & \multirow{4}{*}{$\begin{array}{l}\text { Etapa cujo objetivo foi elaborar o } \\
\text { cenário de avaliação e todos os } \\
\text { artefatos utilizados para a execução } \\
\text { do estudo }\end{array}$} & P.1. Preparação do conteúdo \\
\hline & & $\begin{array}{l}\text { P.2. Montagem do Ambiente de Avali- } \\
\text { ação }\end{array}$ \\
\hline & & P.3. Definição dos Participantes \\
\hline & & $\begin{array}{l}\text { P.4. Construção dos formulários de } \\
\text { avaliação }\end{array}$ \\
\hline \multirow{2}{*}{ 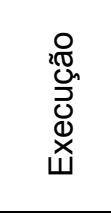 } & \multirow{2}{*}{$\begin{array}{l}\text { Realização do estudo, com atividade } \\
\text { em laboratório. Os alunos foram } \\
\text { monitorados sob supervisão do } \\
\text { professor da disciplina }\end{array}$} & $\begin{array}{l}\text { E.1. Realização da avaliação junto } \\
\text { com os participantes }\end{array}$ \\
\hline & & $\begin{array}{l}\text { E.2. Observação pelo observador da } \\
\text { avaliação }\end{array}$ \\
\hline \multirow{2}{*}{ 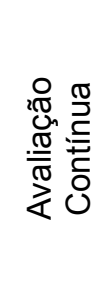 } & \multirow{2}{*}{$\begin{array}{l}\text { Coleta e análise dos aspectos } \\
\text { motivacionais ao longo do curso }\end{array}$} & $\begin{array}{l}\text { A.C. } 1 \text { Coleta dos dados por meio de } \\
\text { formulários de avaliação de aprendiza- } \\
\text { do e satisfação de uso. }\end{array}$ \\
\hline & & $\begin{array}{l}\text { A.C.2. Análise e avaliação da contribu- } \\
\text { ição do MACteaching para o aprendi- } \\
\text { zado dos alunos. }\end{array}$ \\
\hline
\end{tabular}

Adicionalmente, foram elaborados os seguintes documentos e formulários: o Termo de Consentimento Livre e Esclarecido (TCLE) para garantir a segurança dos dados dos participantes; Formulário de Caracterização para identificar o grau de conhecimento e experiência dos participantes em IHC e no uso do MAC e; os Questionários e Formulários Pós-Avaliação para coletar a experiência de uso e percepção de aprendizado dos alunos após o teste. Como infraestrutura para realização do estudo foi escolhido um dos laboratórios de uma Instituição de Ensino Superior do norte do país.

Execução: O estudo foi realizado, no contexto da disciplina de IHC, teve a participação de um observador, responsável pelo desenvolvimento do jogo, responsáveis por observar as dificuldades de interação dos usuários com o aplicativo. E também o professor da disciplina, que foi responsável por supervisionar o estudo aplicado.

Para a avaliação inicial do jogo, nove participantes se disponibilizaram voluntariamente em avaliar MACteaching. Após o uso do aplicativo, foram feitas 
entrevistas e aplicados questionários de avaliação sobre a experiência de uso e aprendizado dos participantes com o MACteaching. Os resultados foram agrupados em pontos fortes, pontos a serem melhorados e a percepção do participante ao usar o aplicativo como ferramenta de apoio ao ensino do Método de Avaliação de Comunicabilidade.

Resultados Obtidos: Para obter os resultados qualitativos foram aplicados questionários após as aulas, onde foi passado o aplicativo para os alunos usarem como ferramenta complementar de aprendizado. Os resultados foram analisados com base nos conceitos do MAC, apenas para a contribuição dos conceitos das etiquetas aplicados no método, conforme apresentado na Tabela 3.

Tabela 3. Resultados Obtidos

\begin{tabular}{|c|c|c|c|c|c|c|c|c|}
\hline Categorias & ID & Questões & 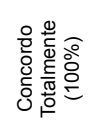 & 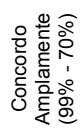 & 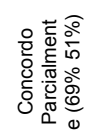 & 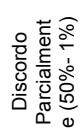 & 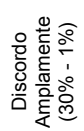 & 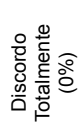 \\
\hline \multirow{6}{*}{ 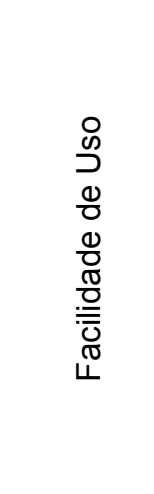 } & F1 & $\begin{array}{l}\text { Foi fácil aprender a utilizar o jogo } \\
\text { MACTEACHING }\end{array}$ & 3 & 4 & 1 & & 1 & \\
\hline & $\mathrm{F} 2$ & $\begin{array}{l}\text { Consegui utilizar o jogo } \\
\text { MACTEACHING da forma que eu } \\
\text { queria }\end{array}$ & 1 & 5 & 2 & 1 & & \\
\hline & F3 & $\begin{array}{l}\text { Eu entendia o que acontecia na minha } \\
\text { interação com a MACTEACHING }\end{array}$ & 4 & 2 & 2 & 1 & & \\
\hline & F4 & $\begin{array}{l}\text { Foi fácil ganhar habilidade no uso da } \\
\text { MACTEACHING }\end{array}$ & 3 & 3 & 3 & & & \\
\hline & F5 & $\begin{array}{l}\text { É fácil lembrar como utilizar o jogo } \\
\text { MACTEACHING }\end{array}$ & 5 & 2 & 2 & & & \\
\hline & F6 & O jogo MACTEACHING é fácil de usar & 4 & 2 & 2 & 1 & & \\
\hline \multirow{3}{*}{$\begin{array}{l}\frac{0}{0} \\
\frac{\pi}{0} \\
: \frac{\bar{T}}{J}\end{array}$} & U1 & $\begin{array}{l}\text { O jogo MACTEACHING me permitiu } \\
\text { aprender mais rápido do que sem } \\
\text { usar o jogo }\end{array}$ & 3 & 4 & 1 & 1 & & \\
\hline & $\mathrm{U} 2$ & $\begin{array}{l}\text { Usar a MACTEACHING melhorou o } \\
\text { meu desempenho na disciplina }\end{array}$ & & 6 & 3 & & & \\
\hline & U3 & $\begin{array}{l}\text { Eu considero a MACTEACHING útil } \\
\text { para aprender o Método de Avaliação } \\
\text { de Comunicabilidade }\end{array}$ & 4 & 5 & & & & \\
\hline \multirow{2}{*}{ 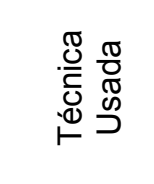 } & T1 & $\begin{array}{l}\text { As rupturas do jogo MACTEACHING } \\
\text { são fáceis de compreender }\end{array}$ & 3 & 2 & 4 & & & \\
\hline & $\mathrm{T} 2$ & $\begin{array}{l}\text { As rupturas do jogo MACTEACHING } \\
\text { utilizam palavras de fácil } \\
\text { entendimento }\end{array}$ & 3 & 5 & & 1 & & \\
\hline
\end{tabular}

Os resultados apresentados na Tabela 3 mostram que a aceitação do jogo foi substancialmente positiva. Em relação a facilidade de uso, $78 \%$ dos participantes concordaram em relação ao ganho de habilidade e interação usando aplicativo. Sobre a utilidade do aplicativo, cerca de $87 \%$ dos participantes relataram que o aplicativo pode auxiliar no ensino do MAC, e de conceitos aplicados em IHC e Engenharia de Software. Adicionalmente, em relação a técnica usada no jogo, de simulação por meio de história, a maioria dos participantes relataram satisfação com a narrativa usada no jogo

No entanto, alguns pontos foram levantados pelos participantes como os levantados pelos participantes 1 e 4 respectivamente, que relataram: "O jogo possui as 
etiquetas confusas, talvez fosse interessante colocar uma contextualização sobre as etiquetas antes da história" e "Acredito que não fica claro os conceitos apresentados sobre os problemas, indicando a relação das rupturas com as etiquetas".

Outro ponto relatado foi relacionado a interatividade do aplicativo, com pontos de customização com sons e novas etiquetas, como apresentado pelo participante 2 , que sugeriu a seguinte melhoria: "Acrescentaria novas músicas e abriria espaço para receber sugestões de novos problemas de comunicabilidade.". Assim como o participante 5 que relatou "Adicionar novas questões para desafiar os outros colegas".

Além disso, o jogo ainda apresentou erros, ocasionados por falhas na construção do aplicativo. Tais erros foram relatados pelos participantes, que apresentaram argumentos como o participante 6 que sugeriu: "Falta de prevenção de erro, quando o jogo trava é perdido a pontuação do recorde do momento."

\section{Análise e Dificuldades}

Como limitador, pode-se destacar a falta de monitoramento aos outros discentes que não responderam aos questionários, para pudéssemos avaliar a captura suas percepções e dificuldades para enriquecer os dados. Além disso, a validade de um estudo está voltada ao grau de confiança que se pode ter no processo de exploração experimental como um todo [Travassos et al., 2002]. Assim, como em outros estudos experimentais existem ameaças, este estudo também apresenta. As ameaças relacionadas a este estudo são classificadas em quatro categorias: Validade Interna, Validade externa, Validade de construção e Validade de Conclusão.

- Validade Interna: nesta foram consideradas as seguintes ameaças: (1) Classificação dos participantes, que foram estudantes. Para o tratamento desta ameaça foram desenvolvidos formulários: TCLE, Caracterização do Participante e Questionário, pois ao assinarem o TCLE o participante ficava ciente do monitoramento e gravações feitas durante o estudo e isso de certa forma influência no comportamento do mesmo. $\mathrm{O}$ tratamento dessa ameaça se deu através de perguntas não definidas feitas pelo pesquisador durante o preenchimento dos formulários, de modo que o participante se familiarizasse com o estudo e se sentisse à vontade para responder aos formulários.

- Validade Externa: foram consideradas as seguintes ameaças: (1) os participantes do estudo foram estudantes de graduação em Engenharia de Software e não profissionais da área de IHC. Mesmo sendo estudantes de graduação os participantes possuíam experiência em desenvolvimento de aplicações móveis. Outra ameaça foi (2) à utilização de ambiente de treinamento ou ensino pode não representar o ambiente industrial. Para tratamento desta foi verificado através do formulário de Caracterização se os participantes haviam publicado alguma aplicação móvel. Apesar de não representar todo o nicho de desenvolvedores, os participantes representam um perfil de novatos.

- Validade de Construção: nesta ameaça foi considerado: (1) o tipo de análise, (2) escala de likert utilizada. Em relação à questão (1) é necessária a utilização de alguma técnica específica para avaliação qualitativa. Sobre a questão (2) a escala de likert utilizada é adotada em estudos que avaliam 
aceitação de tecnologias pelos usuários.

- Validade de Conclusão: para este tipo de ameaça foi considerado o tamanho da amostra, como os participantes são alunos de graduação podem não ser adequados para simular o ambiente industrial. Assim, o tamanho da amostra limita a generalização dos resultados obtidos. Apesar do número relativamente pequeno, estudos afirmam que é possível ter um resultado satisfatório na avaliação de uma tecnologia de software [Coutinho et al. 2014], [Ferreira et al. 2014], [Valentim et al. 2014],[Bim et al. 2011].

\section{Considerações Finais e Trabalhos Futuros}

O presente artigo apresentou o processo de criação e avaliação de um aplicativo, em formato de jogo para o ensino de um dos importantes métodos de IHC, fundamentados na Engenharia Semiótica, o Método de Avaliação de Comunicabilidade (MAC). Como avaliação inicial, apresentada nesse artigo, foi adotada uma avaliação experimental aplicado em ambiente de ensino, em uma disciplina de IHC, no curso de Sistemas de Informação em uma universidade do norte do Brasil.

O estudo foi de extrema importância, pois auxiliou na detecção de rupturas que permitiram o aprimoramento tanto da interface gráfica, como os fluxos de interação do usuário com o aplicativo. Outro ponto importante a ser destacado, está no fato da realização da avaliação na fase de desenvolvimento do aplicativo, o que permitiu a correção das interfaces gráficas e ainda a identificação de novas funcionalidades a serem desenvolvidas e adicionadas ao aplicativo MACteaching.

Os resultados quantitativos se mostraram úteis para a identificação de melhorias. Porém a realização de entrevistas sobre a experiência de uso e aprendizado em sala de aula tornou melhor a compreensão sobre como os usuários utilizam o MACteaching, ao presenciar eventuais dificuldades. Por essa razão, a análise qualitativa foi determinante para entender o comportamento e dificuldades dos participantes.

Com esta pesquisa, espera-se contribuir com a área e o ensino de IHC, assim como disponibilizar o jogo para utilização em salas de aula. Os trabalhos futuros incluem (1) adicionar ao jogo outras atividades do MAC, como as atividades de preparação e coleta de dados; (2) aumentar a quantidade e diversidade de perguntas; (3) incluir mais fases ao jogo e (4) e possibilitar uma maior interação de alunos e professores através do jogo. O MACteaching está disponível na loja de aplicativos ${ }^{1}$

\section{Referencias}

Barbosa, J.D.S. Silva, S.B. (2010). Interação Humano - Computador. 1ra ed. Elsevier, Rio de Janeiro, 2010, pag 385.

Bim, S.A. Prates, R.O. Silveira, M.S. andWinckler, M. (2011) "Ensino de IHCAtualizando as Discussões sobre a Experiência Brasileira". In: Anais do XIX Workshop sobre Educação em Computação (WEI). SBC, Porto Alegre.

Cardoso, L. and Sieckenius, C. (2007). Commest - Uma ferramenta de apoio ao Método de Avaliação de Comunicabilidade. In: III Conferência Latino-Americana de Interação Humano-Computador.

\footnotetext{
${ }^{1}$ https://play.google.com/store/apps/details?id=macapp.ufam.icet.com.mac
} 
Coutinho, W. Couto, E. Biase, C. Fernandes, P. S. and Bonifacio, B. A. (2015) "Improving na Educational Mobile Application Through Usability Evluation. In: Proceedings of International Technology" In: Education and Development Conference, 2015. Madrid, Spain, v. 1. p. 5812-5820.

de Souza, C. S. (2005) The Semiotic Engineering of Human-Computer Interaction. Cambridge, MA: The MIT Press.

Ferreira, B. M.; Rivero, L.; Lopes, A. C.; Marques, A. B.; Conte, T. U. (2014) Apoiando o Ensino de Qualidade de Software: Um Serious Game para o Ensino de Usabilidade. In: Congresso Brasileiro de Software: Teoria e Prática - VII FÓRUM DE EDUCAÇÃO EM ENGENHARIA DE SOFTWARE, 2014, Maceió. Anais do XXVIII Simpósio Brasileiro de Engenharia de Software (SBES 2014). Porto Alegre: SBC, 2014. v. 2. p. 12-21.

Meireles, M.C. and Bonifácio, B. A. (2015) "Uso de Métodos Ágeis e Aprendizagem Baseada em Problema no Ensino de Engenharia de Software: Um Relato de Experiência." In: XXVI Simpósio Brasileiro de Informática na Educação, 2015, Maceió, 2015. p. 180-189.

Mitchell, Alice; Savill-Smith, C. (2004) "The use of computer and video games for learning: A review of the literature." In: Learning and Skills Development Agency (LSDA). pp 12- 25 San Francisco. 2015.

Prates, R.O and Barbosa, S.D.J. (2007) "Introdução à Teoria e Prática da Interação Humano- Computador fundamentada na Engenharia Semiótica”. In T. Kowaltowski \& K. Breitman (orgs) Jornadas de atualização em Informática.

Salgado, C.C.L., Bim, A.S., and Souza, S.C. ( 2006) “Comparação entre os Métodos de Avaliação de Base Cognitiva e Semiótica" Simpósio Brasileiro sobre Fatores Humanos em Sistemas Computacionais (IHC), pp. 158 - 167.

Savi, R. and Ribas, V. (2008) "Jogos Digitais Benefícios e Desafios. Centro Interdisciplinar de Novas Tecnologias na Educação. RENOTE, v. 6, n. 1, 2008.

Shimada, H. e Kitajima, M. (2006) "Why Do Illustrations Promote Text Comprehension? Motivation Effect and Elaboration Effect." In: 5th International Conference of the Cognitive Science. Vancouver, British Columbia, Canada.

Sommariva, L. Benitti, F. B.V. and Dalcin, F.S. (2011). "UsabilityGame: jogo simulador para apoio ao ensino de usabilidade". In: Proceedings of the 10th Brazilian Symposium on Human Factors in Computing Systems and the 5th Latin American Conference on Human-Computer Interaction. Brazilian Computer Society, 2011. p. 61-65.

Valentim, N. M. C.; Rabelo, J.; Silva, W.; Coutinho, W.; Conte, T. (2014). Avaliando a qualidade de um aplicativo web móvel através de um teste de usabilidade: um relato de experiência. In: Simpósio Brasileiro de Qualidade de Software, 2014, Blumenau. XIII Simpósio Brasileiro de Qualidade de Software, 2014. p. 255-263. 